\title{
Fully Isolated Quad-Port Electric Energy Routing Converter with Medium- and High-Voltage Grid Connection Capability
}

\author{
Guanglin Sha, ${ }^{1}$ Qing Duan, ${ }^{1}$ Wanxing Sheng, ${ }^{1}$ Yao Zhang, ${ }^{1}$ Chunyan Ma, ${ }^{1}$ Caihong Zhao, \\ Yan Zhao, ${ }^{1}$ Zhe Liu, ${ }^{2}$ and Jiaxun Teng $\mathbb{D}^{1}$ \\ ${ }^{1}$ China Electric Power Research Institute, Haidian District, Beijing 100192, China \\ ${ }^{2}$ State Grid Shanghai Electric Power Research Institute, Handan Road No. 171, Shanghai 200437, China \\ Correspondence should be addressed to Jiaxun Teng; tengjiaxun@qq.com
}

Received 11 October 2020; Revised 6 November 2021; Accepted 24 November 2021; Published 13 January 2022

Academic Editor: Yuanxin Zhou

Copyright (C) 2022 Guanglin Sha et al. This is an open access article distributed under the Creative Commons Attribution License, which permits unrestricted use, distribution, and reproduction in any medium, provided the original work is properly cited.

\begin{abstract}
Aiming at the demand for medium- and high-voltage port access capability in energy router, this study proposes a quad-port DC/ DC converter topology scheme based on modular multilevel converter (QP-M $\left.{ }^{2} \mathrm{DC}\right)$. Compared with the traditional multiterminal energy routing converter, it has the advantages of high modularity, strong flexibility, and high power density. In addition, for the modular structure on the medium- and high-voltage sides, this study proposes a narrow phase-shift cyclic modulation strategy, which reduces the system need for voltage balance control and simplifies the overall system control. This study comprehensively introduces and analyses the QP-M ${ }^{2} \mathrm{DC}$ topology, working principle, high-frequency link equivalent, and power characteristics, then establishes an equivalent model of system control, and proposes a control scheme for a multiterminal energy routing converter. Finally, a simulation model of the system is established through PLECS, and the simulation results show that in such a narrow phase-shift modulation strategy, the proposed topology can have stable operation in a variety of patterns, reduce the capacitance, and achieve better voltage balance at the same time. The experimental results show the converter efficiency of up to $97.8 \%$. It further shows the superiority of the proposed topology structure and the correctness and effectiveness of the proposed control schemes.
\end{abstract}

\section{Introduction}

With the development of microgrid technology, medium- and high-voltage DC microgrid (DCMG) has become the main research hotspots now. Among them, the medium- and high-voltage DC distribution system is a key link in the establishment of modern DCMG. The distributed power sources in the microgrids include photovoltaics, wind turbines, fuel cells, and energy storage units, and the loads also include a variety of DC loads and AC loads [1]. Due to the diversification of its usage scenarios, multi-bus power distribution structure is required, and its interface types can be a variety of distributed energy sources, energy storage systems, AC and DC loads, etc. [2]. Therefore, in the DCMG energy management system, as an important part of the electrical energy router, it can control the bidirectional flow of energy and realize the voltage conversion and electrical isolation between the medium-voltage direct current (MVDC) and the low-voltage direct current (LVDC). The core device for plug and play is the DC transformer (DCT).

The energy scheduling in DCMG requires multidirectional electric energy routing equipment. To ensure the bus voltage stability and continuous operation of DCMG, it is necessary to configure appropriate energy storage units and bidirectional converters. When excess power is generated inside the microgrid, the extra energy is fed back to the grid, and more energy is obtained from the grid when needed. This requires the design of multiterminal energy routing devices in the DCMG distribution system. Therefore, to facilitate the connection of multiple new energy sources and energy exchange between power grids, multi-port energy routing schemes based on different usage scenarios have been proposed [3-8]. Reference [3] introduced an energy routing topology for wind storage grid-connected system, 
using a full-bridge power converter to implement an independent hybrid renewable energy DC grid system with battery storage. Two different converter structures were proposed in $[4,5]$ for household energy routing equipment, including residential microgrid topology consisting of photovoltaic and fuel cell multi-port DC/DC converters and single-phase grid-connected bidirectional inverters, and the topology using a multi-winding high-frequency transformer to connect each $\mathrm{H}$-bridge port for isolation and power redistribution. Liu et al. [6] proposed a solid-state transformer topology based on quad-active bridge (QAB), including the power transmission analysis, modelling, and control strategy of $\mathrm{QAB}$ and presented a new equivalent model of multiwinding transformer. Frutos et al. [7] designed a linear quadratic regulator controller for a quad-port converter based on the average equivalent model of $\mathrm{QAB}$, and the control strategy can resist step disturbance. The abovementioned topology structure can realize the energy routing function through a reasonable control scheme, but it does not have the medium- and high-voltage access capability.

The dual active bridge (DAB) serves as a basic dc transformer, and it is generally regarded as the core circuit of the next generation of high-density power conversion systems [8]. However, due to the power capacity limitations of power electronic switches, an independent $\mathrm{DAB}$ unit cannot be used as a permanent DCT between the MVDC bus and the LVDC bus of the DCMG. Therefore, to improve the power capacity and voltage level of DCT, the structure of input series and output parallel based on $\mathrm{DAB}$ is a common scheme for DCT to connect MVDC and LVDC [9]. However, this topology also has reliability problems. When a fault occurs, the DC capacitor of the DAB should be recharged after the fault is eliminated, which will slow down the recovery process of current fault. In addition, the existence of DC capacitors in the system makes it difficult to implement the redundancy scheme. When a submodule (SM) fails, the DCT must stop working, which will reduce the reliability of the system. Therefore, in the study of the DC/DC conversion topology scheme with the introduction of medium- and high-voltage ports, the DC converter based on the modular multilevel converter (MMC) structure has also begun to attract the attention of some scholars. Li et al. [10] made a systematic comparison between multilevel high-frequency link dc transformer (abbreviated MDCT) based on modular multilevel converter and multilevel dc link dc transformer (abbreviated ADCT) based on DAB for MVDC application. MDCT uses MMC structure to increase the voltage level, compared with ADCT still using DAB structure as the basic unit, although MDCT requires more switches and highfrequency inductance on the high-voltage side of the same voltage level, it can reduce the number of high-frequency transformers. In addition, the number of $\mathrm{H}$-bridges on the LVDC side of MDCT is only determined by the power level, which can be less than the number of SMs on the MVDC side. However, the ADCT has poor flexibility because the number of modules on the low-voltage side is exactly the same as that on the medium-voltage side [11-13] provided a phase-shift modulation strategy for MMC-based dc converters, but this modulation strategy will cause imbalance of
SM voltage, which requires the additional introduction of voltage balance control strategy. Shao et al. [14] proposed a capacitor voltage balance control strategy that does not rely on arm current detection by introducing a small delay time into the switch pulse of SMs. Sun et al. [15] proposed an optimized dual-phase-shift voltage balance control strategy and adopted NLM algorithm for the high-frequency switch controlled by voltage balance.

Therefore, the topology of the multi-port energy routing scheme for most current uses is the full-bridge structure. It uses multi-winding transformer to integrate multiple fullbridge structure, but there is current recovery after the failure recovery is slow and redundancy scheme is difficult to realize. The modular structure of multilevel converter has the advantage of flexibility [16-23]. In this study, a four-port converter is constructed. The control mode of multi-port converter is mainly based on phase-shift control, and the voltage balance control strategy is introduced to solve the voltage imbalance caused by phase-shift control or optimize the phase-shift control and put forward a kind of doublephase-shift voltage balance control strategy. This study puts forward a kind of narrow phase-shift modulation strategy and reduces the influence of the voltage unbalance of converter.

Based on the above analysis, this study proposes a topology scheme of quad-port DC/DC converter based on modular multilevel structure to realize the multiterminal power routing transformation with medium- and high-voltage port access in the DCMG multi-bus distribution application. This topology integrates the advantages of $M M C$ and traditional $\mathrm{DAB}$, with a high-voltage working level and flexible modular design. In addition, when the MMC structure on the medium-voltage side works in high-frequency quasi-square-wave modulation, the SM can meet the requirements using thin-film capacitance with a small capacitance value, which improves the power density of the system. Compared with the traditional multiport energy routing schemes, higher reliability exists in the proposed converter. It not only meets the needs of high power capacity but also uses the film instead of electrolytic capacitors. The converter has small internal energy and high power density. The proposed narrow phase-shift cyclic modulation strategy avoids the problem of voltage unbalance control strategy in traditional phase-shift-down control scheme and simplifies the control.

In this study, the topological structure, working principle, equivalent analysis of high-frequency link (HFL), and power transfer characteristics of QP- $\mathrm{M}^{2} \mathrm{DC}$ are comprehensively introduced and analysed, the equivalent model of system control is established, and a control scheme of multiterminal energy routing converter is proposed. In addition, a kind of MMC side narrow phase-shift cyclic modulation strategy is proposed, which reduces the need for equilibrium control and simplifies the overall control of the system. Finally, the simulation model of $\mathrm{QP}-\mathrm{M}^{2} \mathrm{DC}$ is established, and the simulation results verify the correctness and effectiveness of the proposed scheme. At the same time, an experimental platform is set up, and the maximum efficiency of the proposed scheme can reach $97.8 \%$, which further verifies the superiority of the scheme. 


\section{Operating Characteristics of Proposed QP-M $\mathbf{M}^{2} \mathbf{D C}$}

2.1. QP- $M^{2} D C$ Topology. The QP-M ${ }^{2} \mathrm{DC}$ topology proposed in this study is shown in Figure 1 The QP- ${ }^{2}$ DC topology consists of an MVDC port transform module, quad-port high-frequency link, and three LVDC port transform modules. The MVDC interface of the topology is composed of modular multilevel structure. Each phase of the bridge arm is composed of a semibridge $\mathrm{SM}_{\mathrm{i}}$ and a bridge arm inductor in series, and each semibridge module contains an SM capacitance. The voltage balance of SMs is the prerequisite for the MMC to modulate the ideal voltage, and it is also the key to the stable operation of the system. The HFL consists of a multi-port high-frequency transformer and a power inductor to transfer energy. The LVDC port consists of an H-bridge, and the energy storage port includes a halfbridge buck/boost, which ensures bidirectional transmission of energy at the low-voltage port.

Compared with the traditional multilevel $\mathrm{DAB}$ cascade structure, the proposed QP- $\mathrm{M}^{2} \mathrm{DC}$ topology scheme adopts MMC structure to realize the connection function of medium and high voltage, which meets the demand of high power capacity. Compared with $\mathrm{DAB}$ cascade structure, the proposed topology uses thin-film capacitor with small capacitance value to replace electrolytic capacitance, which has the advantage of small internal energy storage in the converter. In the application of DCMG power distribution in low and medium power levels, traditional DAB cascade structure to meet the voltage level of medium- and highvoltage ports, the traditional $\mathrm{DAB}$ cascade structure has the same number of transformers as that of medium- and highvoltage sides, which is not conducive to the design of power density improvement of the system. The topology of the MMC in this study is based on voltage level as standard for MMC on the high-voltage side and current level as standard on multilevel power module on the low-voltage side. There is no mutual restriction on the number of modules in the twostage design. It has more flexibility, which also contributes to the improvement of system power density.

In Figure 1, $V_{a}$ and $I_{a}$, respectively, represent the voltage and current of the high-voltage side. $V_{b}, V_{c}, V_{d}, i_{b}, i_{c}$, and $i_{d}$, respectively, represent the output voltage and current of the three ports on the low-voltage side. $V_{a j}$ and $i_{a j}$ are the voltage and current of MMC bridge arm. $L_{a}, L_{b}, L_{c}$, and $L_{d}$ are HFL phase-shift inductors. An equivalent analysis of high-frequency link phase-shift inductor will be carried out later.

2.2. MMC Modulation Strategy. The topology is divided into MVDC side and LVDC side, and the MVDC side is composed of MMC structure. There are many modulation strategies for the MMC. Reference [24] adopted a carrier phase-shift control (CPS-PWM), as shown in Figure 2(a), and the control signals of the upper and lower bridge arms are obtained by comparing $N$ carrier waves with a phase difference of $2 \pi / N$ with modulated waves of opposite phase. This modulation strategy cannot guarantee the natural balance of the voltage of the SMs in QP-M ${ }^{2} \mathrm{DC}$, and it is difficult to ensure that $N$ SMs are put in each phase at any time after the addition of the balancing strategy, so a large bridge arm buffer inductance is often needed to help suppress loop current between phases. He et al. [25] introduced a carrier stack modulation (CAM-PWM). As shown in Figure 2(b), by superimposing the carrier waves and comparing with the modulated wave, the number of carrier waves that are smaller than the modulated wave is the number of SMs of the upper (or lower) bridge arm, and the output voltages of the modules are superimposed to obtain the output voltage waveform of the upper (or lower) bridge arm. In high-frequency isolated DC/DC transformation, both CPS-PWM and CAM-PWM modulation strategies need high-frequency modulation waves to realize, so the carrier frequency will be higher, which means MMC will work at very high switching frequency, which is difficult to achieve in practical engineering.

Based on the shortcomings of the traditional modulation strategy analysed above in the high-frequency application of MMC, this study proposes a narrow phase-shift cyclic (NPSC) modulation scheme. A small phase-shift angle $\theta$ is given between the control signals of adjacent SMs in the MMC bridge arm. When $\theta$ is small, the MMC bridge arm voltage is a stepped wave similar to the square wave, which can not only achieve the higher voltage level requirements but can also avoid damage to the circuit due to excessive $\mathrm{d} v / \mathrm{d} t$.

Although the control strategy of Figure 3(a) can achieve the requirements of the bridge arm high-voltage level, the fixed difference in the charging and discharging timing of the bridge arm SMs will cause the voltage imbalance. Therefore, on this basis, a NPSC modulation strategy is designed as shown in Figure 3(b), the phase-shift angle of $n$ SMs is set as $\theta, 2 \theta, \ldots, n \theta$ in the first cycle of drive control, and the second cycle changes the phase-shift angle to $n \theta, \ldots$, $2 \theta, \theta$. The NPSC modulation can realize the charging and discharging balance of the capacitances, and the voltage balance of the SM can be realized under the ideal parameter condition of MMC, which reduces the demand of MMC for the SM voltage balance control.

2.3. Phase-Shift Principle. Combined with the HFL design in Figure 1, similar to the analysis of $\mathrm{DAB}$ in power transmission control, each port of the HFL is equivalent to a voltage source, as shown in Figure 4. The flow direction and magnitude of power transmitted between the ports are controlled by the phase-shift angle between the voltage sources. When energy is transferred between the low-voltage ports, the transfer method can be equivalent to a DAB structure, the voltage of each transformer port is a square wave, and the power is transferred through the phase-shift angle between the low-voltage ports, as shown in Figure 5(a). The power transfer formula can be referred to $\mathrm{DAB}$, and the traditional $\mathrm{DAB}$ power transfer formula can be easily obtained [18] as follows:

$$
P_{o}=\int_{0}^{2 \pi} V_{a b} i_{L} \mathrm{~d} \phi=\frac{G V_{a}^{2}}{\pi \omega L}\left(-\phi^{2}+\pi \phi\right),
$$




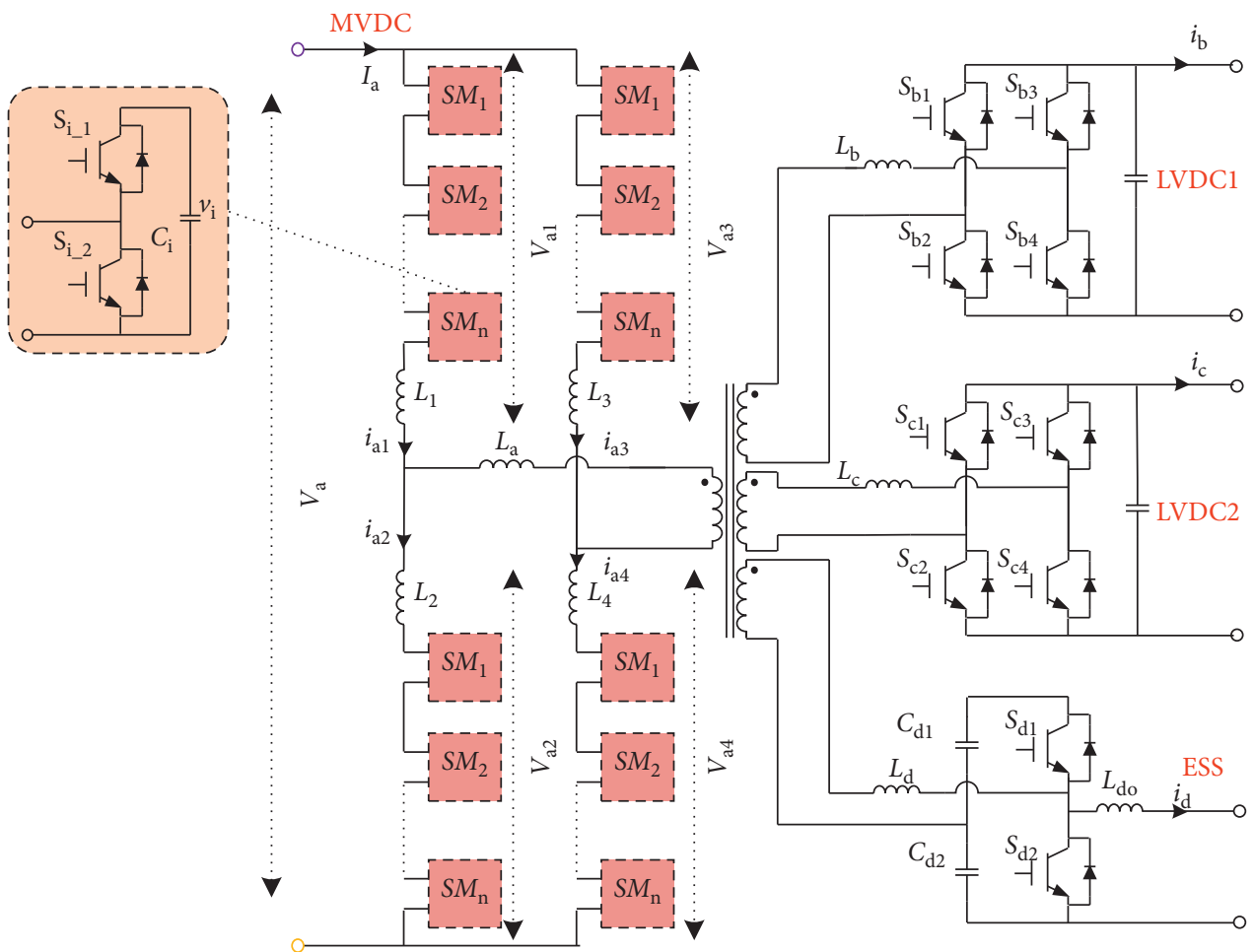

Figure 1: QP-M²DC topology.

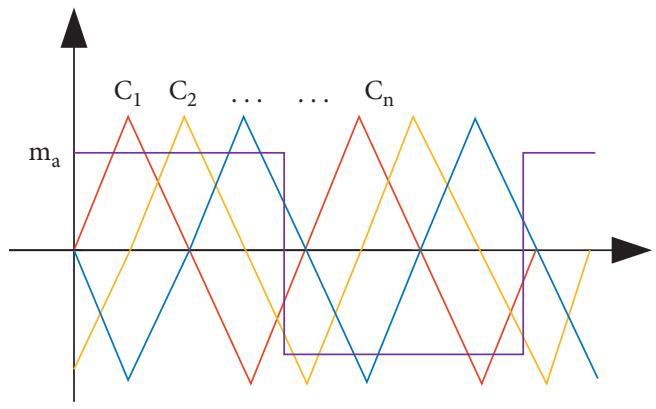

(a)

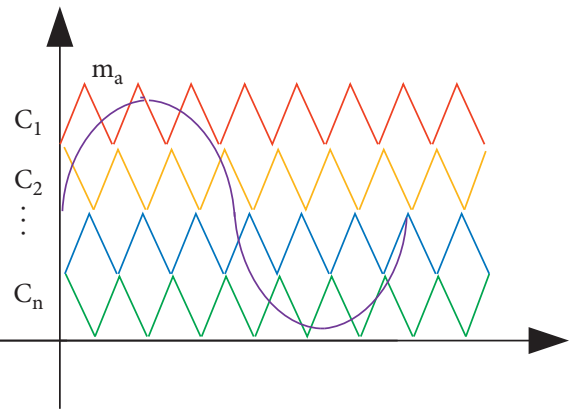

(b)

FIgUre 2: Modulation strategies. (a) CPS-PWM and (b) CAM-PWM.

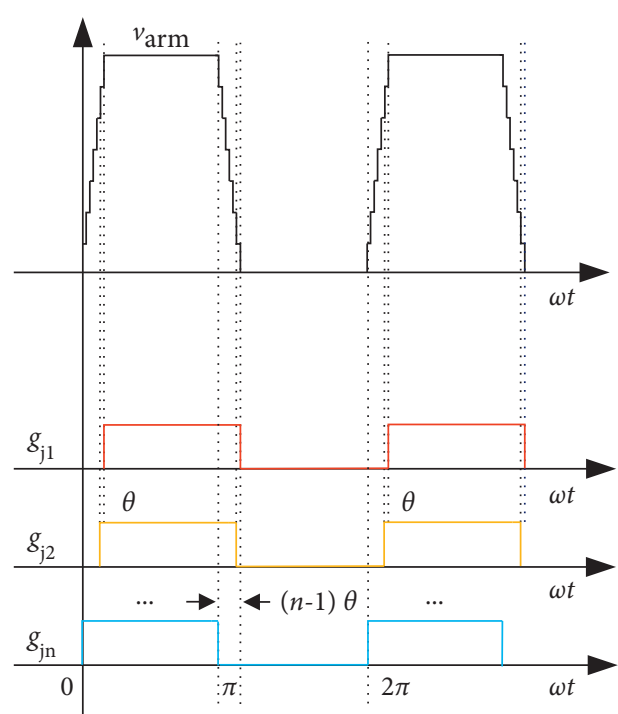

(a)

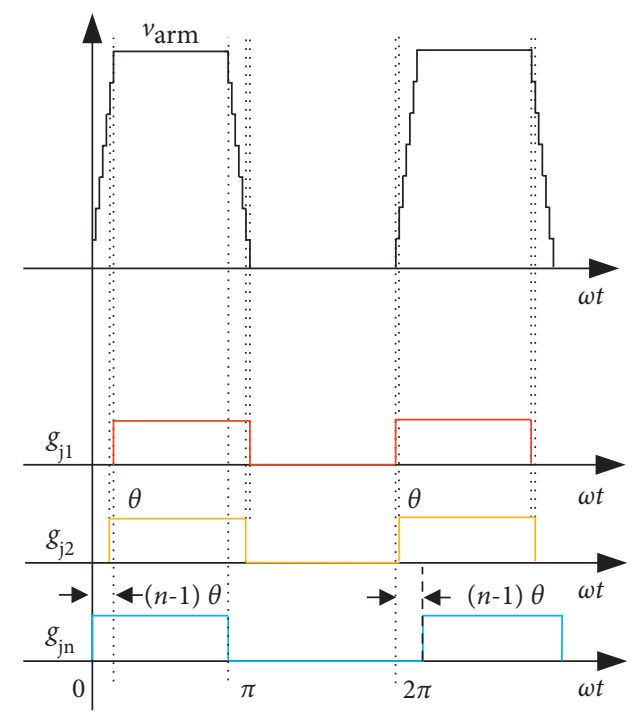

(b)

FIgURe 3: Modulation strategy. (a) NPS and (b) NPSC. 


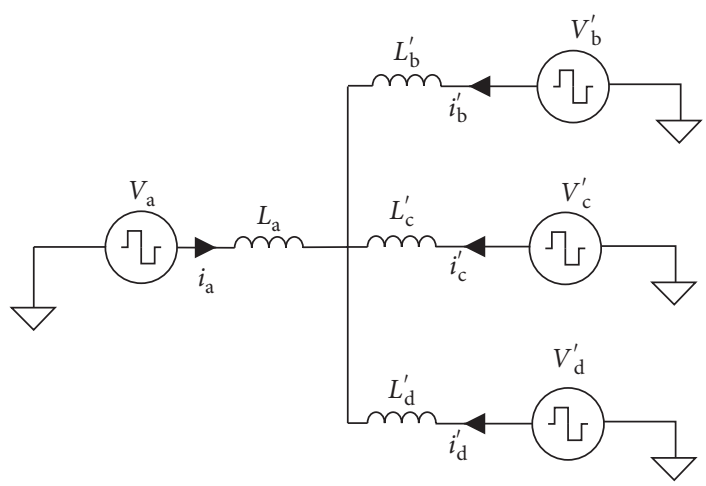

FIGURE 4: Equivalent model of high-frequency link.

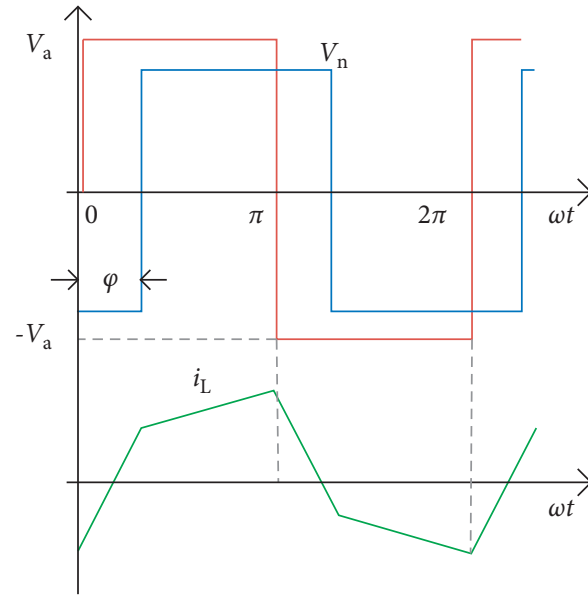

(a)

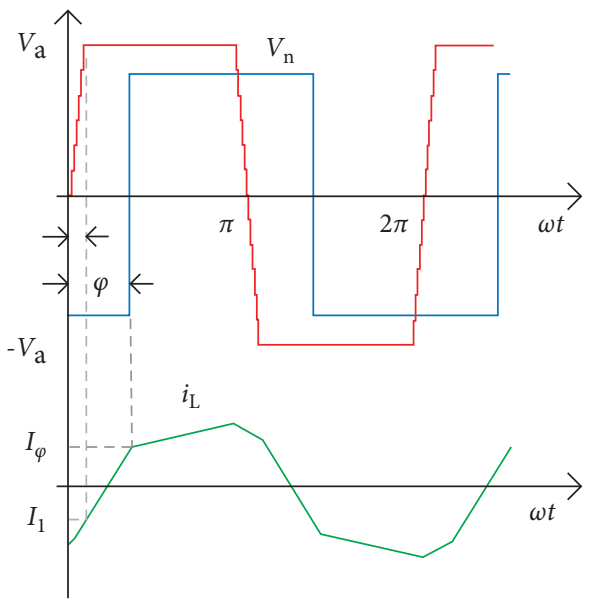

(b)

FIgURE 5: Schematic diagram of working principle. (a) Conventional DAB and (b) MMC-based DAB.

where $\varphi$ is the phase-shift angle between the primary and secondary sides.

When the energy is transferred from the high-voltage side to the low-voltage side, since the high-voltage side MMC structure adopts the NPSC modulation strategy proposed in this study, compared with the traditional DAB structure, a stepped quasi-square wave will be generated on the primary side of the transformer. For the controllable transmission of power between multiple ports, it is necessary to study the applicability of phase-shift control in QP-M ${ }^{2} \mathrm{DC}$ topology. For example, the energy transfer from highvoltage port to any other port is taken, and the waveforms of the inductor current $i_{L}$ and the voltage source are shown in Figure 5(b).

The expression of the MVDC port participating in the power transfer under the NPSC modulation strategy is now derived according to (1). The research scheme considers the situation when $\varphi>(N-1) \theta / 2$, and the formula of inductive current is derived as follows:

$$
\begin{cases}i_{L}\left(\phi_{1}\right)=I_{0}+\frac{1}{\omega L}\left(V_{a}+n V_{n}\right) \phi_{1}, & 0 \leq f_{1}<f, \\ i_{L}\left(\phi_{1}\right)=I_{\phi}+\frac{1}{\omega L}\left(V_{a}-n V_{n}\right)\left(\phi_{1}-\phi\right), & \phi \leq \phi_{1}<\pi-(N-1) \theta, \\ i_{L}\left(\phi_{1}\right)=I_{\pi-m \theta}+\frac{1}{\omega L}\left(V_{a}-\frac{2 m V_{a}}{N}-n V_{n}\right)\left(\phi_{1}-\pi+m \theta\right), & \pi-m \theta \leq \phi_{1}<\pi-(m-1) \theta, m=1,2, \ldots, N-1 .\end{cases}
$$


To simplify the analysis, $\varphi_{1}=\varphi-(N-1) \theta / 2$ is taken, where $V_{1}$ is the voltage amplitude, $n$ is the transformer ratio, $L$ is the phase-shifted inductance converted to the primary side, $\omega=2 \pi f$, and $m$ is the number of steps. From $i_{L}(0)=-i_{L}$ $(\pi)$, we have the following:

$$
\left\{\begin{array}{l}
I_{o}=\frac{V_{a}}{2 \omega L}[-2 G \phi+(N-1) \theta+(G-1) \pi] \\
I_{\phi}=\frac{V_{a}}{2 \omega L}[2 \phi+(N-1) \theta+(G-1) \pi] \\
I_{\pi-m \theta}=\frac{V_{a}}{2 \omega L}\left\{2 G \phi+\left[-\frac{2}{N} m^{2}+\left(-\frac{2}{N}+2 G+2\right) m-N+1\right] \theta+(1-G) \pi\right\}, \quad m=0,1, \ldots, N-1 .
\end{array}\right.
$$

Thus, the expression of MVDC port participating in power transfer under the NPSC modulation strategy can be obtained as follows:

$$
P_{o}=\int_{0}^{2 \pi} V_{a b} i_{L} \mathrm{~d} \phi=\frac{G V_{a}^{2}}{\pi \omega L}\left(-\phi^{2}+\pi \phi-\frac{N^{2}-1}{12} \theta^{2}\right) .
$$

\section{Multiport Energy Transfer Analysis}

3.1. HFL Equivalent Analysis. The MVDC is equivalent to port $a$, and the power transfer between any two ports is mainly accomplished through the phase-shift control of quasi-square-wave voltage. Due to the particularity of the structure of port $a$, the energy transmission is temporarily defined as the transfer of port $a$ to other ports in the HFL equivalent analysis. As shown in Figure 5(b), the squarewave voltage generated by port $a$ is set to $V_{a}$, the current is $i_{a}$, and the inductance is $L_{\mathrm{a}}$. This study does not consider the effect of high-frequency transformer internal leakage inductance. The modulated voltage, current, and inductance at port $k(k=b, c, d)$ are equivalent to port $a$ as follows:

$$
\begin{aligned}
V_{k}^{\prime} & =\frac{N_{a}}{N_{k}} V_{k}, \\
i_{k}^{\prime} & =\frac{N_{k}}{N_{a}} i_{k}, \\
L_{k}^{\prime} & =\left(\frac{N_{a}}{N_{k}}\right)^{2} L_{k} .
\end{aligned}
$$

Port $a$ is selected as the sending port, while port $k(b, c$, and $d$ ) is selected as the receiving ports. According to the equivalent circuit in Figure 4, the total equivalent inductance can be calculated as follows:

$$
L_{\mathrm{THa}}=\left(\sum_{k}^{n} \frac{1}{L_{k}^{\prime}}\right)^{-1} \text {. }
$$

Similarly, the Thevenin equivalent ac voltage $V_{\text {Thk }}$ associated with port $k$ is defined in port $a$ as follows:

$$
V_{\mathrm{THK}}=\frac{\left(\sum_{k}^{n}\left(1 / L_{k}^{\prime}\right)\right)^{-1}}{L_{k}^{\prime}+\left(\sum_{k}^{n}\left(1 / L_{k}^{\prime}\right)\right)^{-1}} \cdot V_{k}^{\prime}
$$

The above is the general equation of the topology with $n$ ports on the low-voltage side. The equivalent circuit can be obtained by combining (6) and (7).

From (4), the power $P_{a k}$ transferred from port $a$ to port $k$ can be obtained as (8), where $\varphi_{\mathrm{ak}}$ is the phase-shift angle between port $a$ and port $k$ :

$$
P_{a k}=\frac{V_{a} V_{\mathrm{THK}}}{\pi \omega\left(L_{a}+L_{\mathrm{THa}}\right)}\left(-\phi_{a k}^{2}+\pi \phi_{a k}-\frac{N^{2}-1}{12} \theta^{2}\right) .
$$

According to Figure 6, the link inductance between the ports can be expressed as follows:

$$
L_{a k}=L_{a}+\left(\sum_{k}^{n} \frac{1}{L_{k}^{\prime}}\right)^{-1} \text {. }
$$

For the average model of this topology, the periodic average current value of the ripple current $i_{a}$ on the DC side of port $a$ is defined as $I_{a}$. The average current generated by port $k$, defined as $I_{a k}$, is given by the following equation:

$$
I_{a k}=\frac{P_{a k}}{V_{a}} .
$$

When $V_{k}^{\prime}=\left(N_{a} / N_{k}\right) * V_{k}$, equations (6)-(9) are substituted into (10), and we get the following equation:

$$
I_{a k}=\frac{P_{a k}}{V_{a}}=\frac{N_{a}}{N_{K}} \frac{V_{K}}{\pi \omega L_{a k}}\left(-\phi_{a k}^{2}+\pi \phi_{a k}-\frac{N^{2}-1}{12} \theta^{2}\right) .
$$

The relevant gain parameter can be calculated as follows:

$$
g_{a k}=\frac{I_{a k}}{V_{K}}=\frac{N_{a}}{N_{K} \pi \omega L_{a k}}\left(-\phi_{a k}^{2}+\pi \phi_{a k}-\frac{N^{2}-1}{12} \theta^{2}\right) .
$$

Therefore, according to the superposition theorem, $I_{a}$ can be expressed as follows:

$$
I_{a}=\sum_{k \neq a}^{n} I_{a k}=\sum_{k \neq a}^{n} g_{a k} V_{k} .
$$




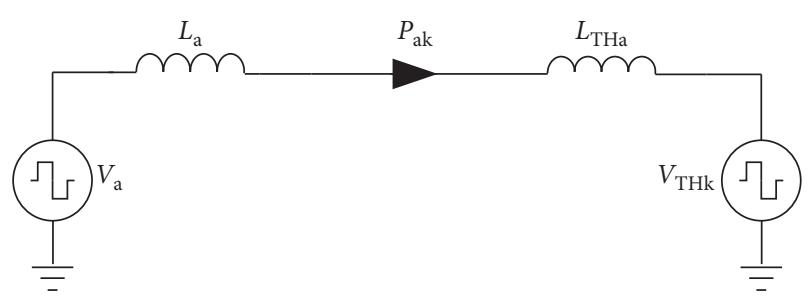

FIGURE 6: Thevenin equivalent circuit between port $a$ and port $k$.

According to the above analysis, the equivalent average model of QP-M ${ }^{2}$ DC topology can be obtained, as shown in Figure 7.

3.2. $Q P-M^{2} D C$ Power Characteristic. To design the rated power of the four ports, the power transfer analysis of the quad-port converter should be carried out based on each possible operating condition. Assuming that in a certain operating condition, there is a port in $\mathrm{QP}-\mathrm{M}^{2} \mathrm{DC}$ operating at the maximum power within the allowable range of phaseshift angle, the rated power of the converter port is limited by the maximum input or output power. Therefore, the rated power of the ports in the quad-port converter depends on the number of sources and loads, as well as the direction of port power flow. The nonlinear relationship is now defined as follows:

$$
\psi(\varphi, \theta)=\left(\pi \varphi-\varphi^{2}-\pi \frac{N^{2}-1}{12} \theta^{2}\right) .
$$

An ideal working mode is defined; that is, each port can carry out bidirectional power transfer. Then, the maximum power transferred from port $\mathrm{j}$ to port $\mathrm{k}$ can be defined according to (8).

$$
P_{j k_{-} \max }=\frac{d_{j} d_{k} V_{a}^{2}}{\pi \omega L_{j k}} \psi(\varphi, \theta),
$$

where $\varphi$ is the phase-shift angle between the two ports, $\theta$ represents the narrow phase-shift angle between the SMs, and this angle can be ignored when the source port is not an MMC structure. $d_{j}=V_{j} / V_{a}$ is the DC conversion ratio of port $j$ and port $a$, and $d_{k}=V_{k} / V_{a}$ is the DC conversion ratio of port $a$ and port $k$. When the $d_{j} d_{k}$ approximates to 1 , the converter can achieve ZVS operation under light loads.

QP-M ${ }^{2} \mathrm{DC}$ can operate in DAB mode by disabling two ports. To further simplify the analysis process, the following assumptions are made: $d_{j}=1, L_{j}^{\prime}=L_{a}$, and then $L_{a k}=L_{a}+L_{a k}=4 L_{a}$. The maximum power that QP-M ${ }^{2} \mathrm{DC}$ flows between any two ports is defined as $P_{\max }$, also known as the maximum link power. It can be obtained as follows:

$$
P_{\max }=\frac{1}{2} P_{\text {DAB_max }} \text {. }
$$

where $P_{\mathrm{DAB}_{-} \text {max }}$ is the maximum power that QP-M ${ }^{2} \mathrm{DC}$ can transfer when working in $\mathrm{DAB}$ mode. Considering the influence of $\theta$ in the control strategy, to simplify the process of power transfer analysis, it is expressed in the form of per unit value.

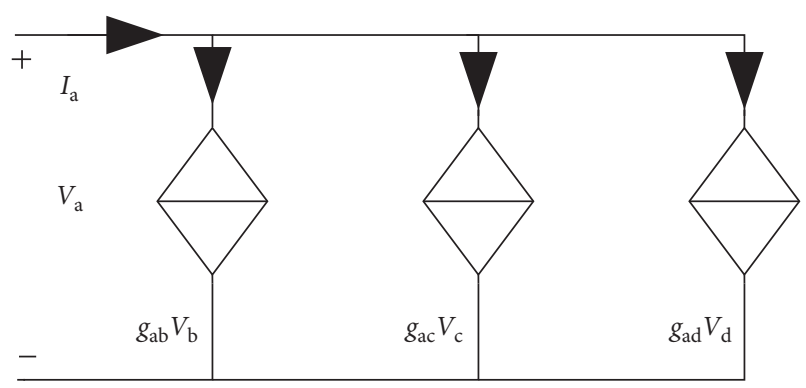

FIGURE 7: DC conversion average model.

$$
\left\{\begin{array}{l}
P_{\text {max } \_ \text {pu }}=\frac{P_{L_{-} \text {max }}}{P_{\text {Base }}}=\frac{1}{2} \psi(\phi, \theta), \\
P_{\text {Base }}=\frac{V_{a}^{2}}{\pi \omega L_{\mathrm{DAB}}},
\end{array}\right.
$$

where $L_{\mathrm{DAB}}=2 L_{a}$, when the phase-shift angle between the source and the load reaches $\varphi$, the source outputs the maximum power. In addition, in QP-M ${ }^{2} \mathrm{DC}$, it is possible to increase the maximum transmission power between the source-load ports by enabling some of the zero-power output ports. The passive ports are hereinafter referred to as zero-power ports, as shown in Figure 8.

Figure 8 depicts the maximum power transfer state when using $r$ sources, $q$ loads, and $p$ zero-power ports, where $\alpha$ is the phase-shift angle between the zero-power ports and the source ports, and $\beta$ is the phase-shift angle between the zero-power ports and the load ports. It can be concluded from Figure 8.

$$
\left\{\begin{array}{l}
\varphi=\alpha+\beta \\
r+p+q=4
\end{array}\right.
$$

Assuming that there is no loss when the power is transmitted between the ports, the following can be obtained:

$$
\begin{aligned}
\frac{r p \psi(\varphi, \alpha)}{2} & =\frac{p q \psi(\varphi, \theta)}{2}, \\
\psi(\beta, \theta) & =\frac{r}{q} \psi(\alpha, \theta), \\
\beta & =\psi^{-1}\left(\frac{r}{q} \psi(\alpha, \theta)\right) .
\end{aligned}
$$

For the special case, when $r=p$, then $\beta=\varphi / 2$, and the maximum link power transferred from the source ports to the load ports is as follows:

$$
\begin{aligned}
P_{\max \_p u} & =\frac{r}{2}(q \psi(\varphi, \theta)+p \psi(\alpha, \theta)) \\
& =\frac{q}{2}(r \psi(\varphi, \theta)+p \psi(\beta, \theta)) .
\end{aligned}
$$

Therefore, when designing the system power transfer level, after adding the zero-power port as a channel for system power transfer, the maximum unit power of each source port is as follows: 


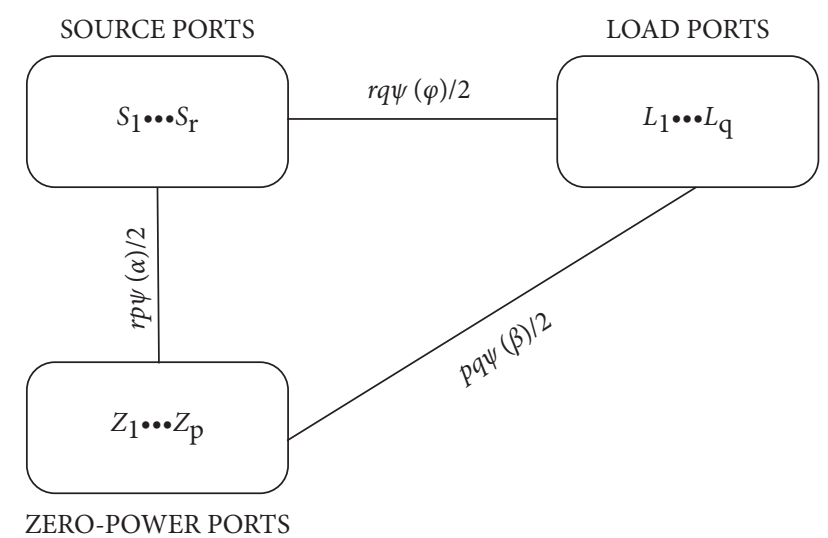

FIGURE 8: QP-M ${ }^{2}$ DC maximum power transmission diagram.

$$
P_{S_{-} \max -\mathrm{pu}}=\frac{1}{2}(q \psi(\varphi, \theta)+p \psi(\alpha, \theta))
$$

Similarly, the maximum unit power of each load port is as follows:

$$
P_{L_{-} \max \_ \text {pu }}=\frac{1}{2}(r \psi(\varphi, \theta)+p \psi(\beta, \theta)) .
$$

3.3. QP-M ${ }^{2}$ DC Power Flow Analysis. According to the above analysis, the average equivalent model of $\mathrm{QP}-\mathrm{M}^{2} \mathrm{DC}$ can be obtained, as shown in Figure 9. When reaching the maximum link power between any two ports of QP-M ${ }^{2} \mathrm{DC}$, the power transfer of the converter depends on the number of source ports, zero-power ports, and load ports.

Figure 10 shows a schematic diagram of the converter working at maximum power, where $\alpha_{d}$ can be solved by (24). When QP-M ${ }^{2} \mathrm{DC}$ works in two-port or three-port state, the zero-power ports automatically participate in the power transmission process.

$$
\phi=\alpha_{d}+\psi^{-1}\left(2 \psi\left(\alpha_{d}, \theta\right)\right) .
$$

The maximum link power of QP-M ${ }^{2} \mathrm{DC}$ can be obtained from (18). When working in one-source one-load (1S-1L) mode, as shown in Figure 3(a), according to (22), the available transmission power is as follows:

$$
P_{1{\mathrm{~S} 1 \mathrm{~L}_{-} \max }_{-} \mathrm{pu}}=\frac{1}{2} \psi(\varphi, \theta)+\psi\left(\frac{\varphi}{2}, \theta\right) .
$$

By the same analysis, the maximum operating power of QP-M ${ }^{2}$ DC in different working modes can be obtained, as shown in Table 1.

\section{Control Strategy Design}

In the power scheduling process of the multi-directional energy routing converter, the characteristics of multiple types of ports need to be considered, such as the characteristics of the photovoltaic port, the multiple energy storage types, and the multi-level power supply bus port. Based on the introduction of the topology structure of QP-M ${ }^{2} \mathrm{DC}$ and the analysis of the power characteristics, this study designs a

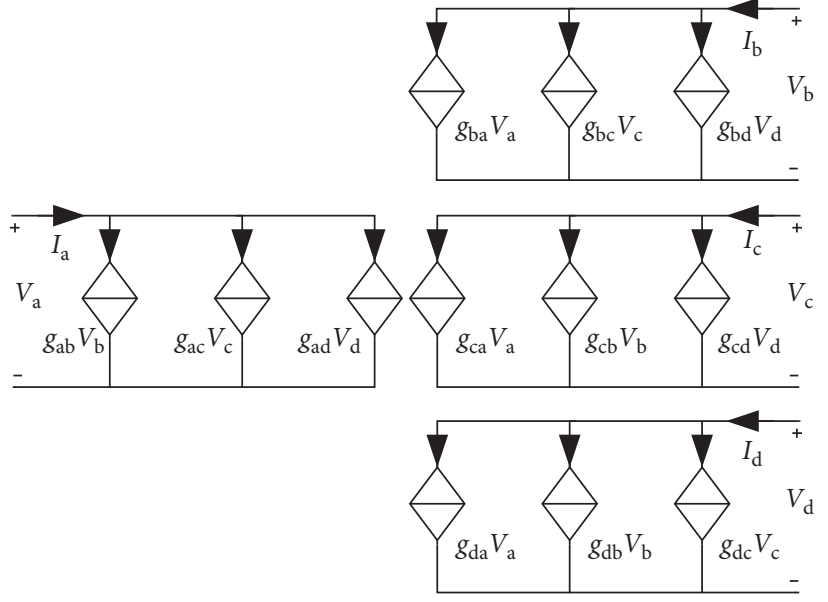

FIgURE 9: Average equivalent model of QP-M $\mathrm{M}^{2} \mathrm{DC}$ converter.

general control strategy for multi-type port power routing, as shown in Figure 11.

The overall control strategy of the energy router of the DC distribution system includes port power control of different interface types, power scheduling control, and phase-shift control strategy. The port power control uses different control strategies according to the characteristics of different devices connected, which may include power flow control of the MVDC grid, maximum power point tracking (MPPT) control of the photovoltaic module, and battery management system (BMS). The power scheduling control is based on the different functions of each port to autonomously schedule power in different applications. The phaseshift control includes the energy transfer control method between the ports and the MMC modulation strategy.

\section{Simulation Results}

Based on the analysis of the QP- $\mathrm{M}^{2} \mathrm{DC}$ power characteristics and the proposed system control strategy under the multimode, this study designs four typical modal simulations and defines the MVDC port as a stable medium-voltage DC bus of $20 \mathrm{kV}$ constant voltage port, LVDC1 and LVDC2 ports are, respectively, $750 \mathrm{~V}$ and $220 \mathrm{~V}$ power supply double bus bars of DCMG, and the ESS port is an energy storage system port. Due to the existence of distributed energy in the DCMG, all four ports are defined as bidirectional energy transfer ports. Simulation parameters are shown in Tables 2 and 3 .

In the control system, a port is set as the reference port with phase angle of 0 . The voltage stability of the reference port needs to be controlled to ensure the stability of the control system, and the voltage or power of the other ports can be controlled by setting the different phase angles with the reference port after power decoupling.

According to the power characteristic analysis, it can be seen that with the increase in the number of ports, the analysis method will become more and more complex when calculating the phase-shift angles, which is difficult to achieve in practical engineering applications. Based on the above analysis, within the allowable error range, voltage, 

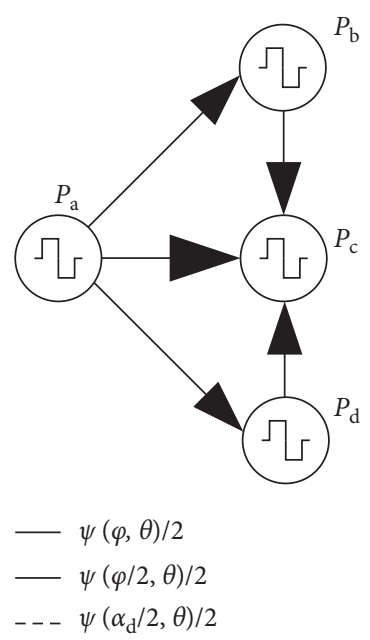

(a)
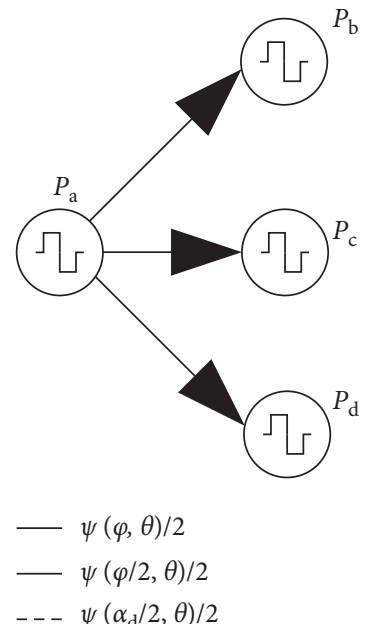

(e)
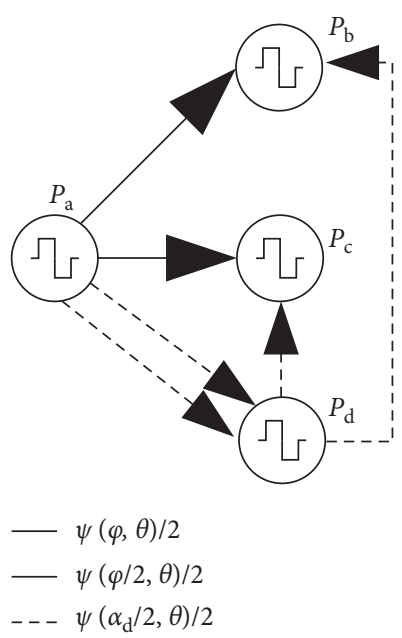

(b)

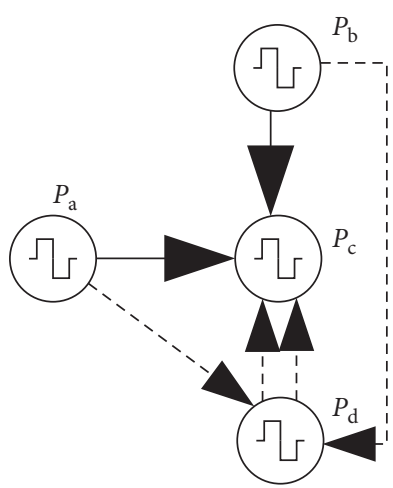

$-\psi(\varphi, \theta) / 2$

$-\psi(\varphi / 2, \theta) / 2$

- - $\psi\left(\alpha_{\mathrm{d}} / 2, \theta\right) / 2$

(c)

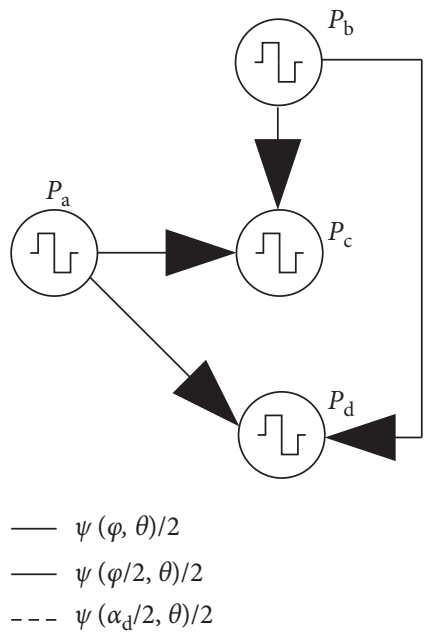

(d)
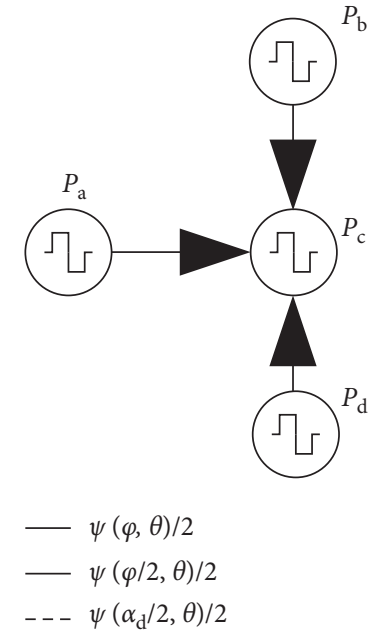

(f)

Figure 10: QP-M²DC maximum power operation mode. (a) 1S-1L, (b) 1S-2L, (c) 2S-1L, (d) 2S-2L, (e) 1S-3L, and (f) 3S-1L.

TABLE 1: Formulas of maximum power in different working modes.

\begin{tabular}{|c|c|}
\hline Mode & Formulas \\
\hline $1 \mathrm{~S}-1 \mathrm{~L}$ & $P_{1 \mathrm{~S}_{1 L_{-}} \max }{ }_{\mathrm{pu}}=(1 / 2) \psi(\varphi, \theta)+\psi((\varphi / 2), \theta)$ \\
\hline $1 \mathrm{~S}-2 \mathrm{~L}$ or $2 \mathrm{~S}-1 \mathrm{~L}$ & 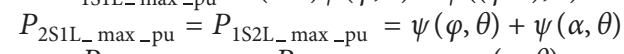 \\
\hline $2 \mathrm{~S}-2 \mathrm{~L}$ & $P_{2 \mathrm{~S}_{2} \mathrm{~L}_{-} \max \_\mathrm{pu}}=P_{\mathrm{DAB}_{-} \max _{-} \mathrm{pu}}=\psi(\varphi, \theta)$ \\
\hline $1 S-3 \mathrm{~L}$ or $3 \mathrm{~S}-1 \mathrm{~L}$ & $P_{1 S_{3 L_{-}} \max \_\mathrm{pu}}=P_{3 \mathrm{~S}_{1 \mathrm{~L}_{-} \max } \mathrm{pu}}=(3 / 2) \psi(\varphi, \theta)$ \\
\hline
\end{tabular}

current with phase-shift angle, or power with phase-shift angle can be regarded as an approximately linear relationship. Therefore, as shown in Figure 12, a combined control scheme based on voltage, current, and power is adopted in the simulation case, and the four ports are bidirectional energy transfer ports, among which MVDC is the gridconnected port of the MVDC bus, it is the reference port of the system, and the voltage is stable, i.e., $\varphi_{0}=0$.

In the simulation case, MMC modulation scheme of MVDC port adopts the NPSC modulation strategy shown in Figure 3(b). As shown in Figure 13, under this quasi-squarewave modulation strategy, when the capacitance of MMC
SM is only $80 \mathrm{uF}$, the voltage fluctuation of the SM capacitor is only $0.7 \%$ and can achieve better voltage balance, which is of great significance to the stable and safe operation of the system. The function can be realized using thin-film capacitor in SM, which greatly reduces the number of electrolytic capacitors used in the system and improves the power density of the energy routing system. Figure 14 shows the modulation voltage waveform on the MMC side. The number of voltage steps is one more than the number of SM, i.e., $N+1$.

Figure 15 shows the disturbance test of the QP-M ${ }^{2} \mathrm{DC}$ converter in the one-source three-load mode with the 


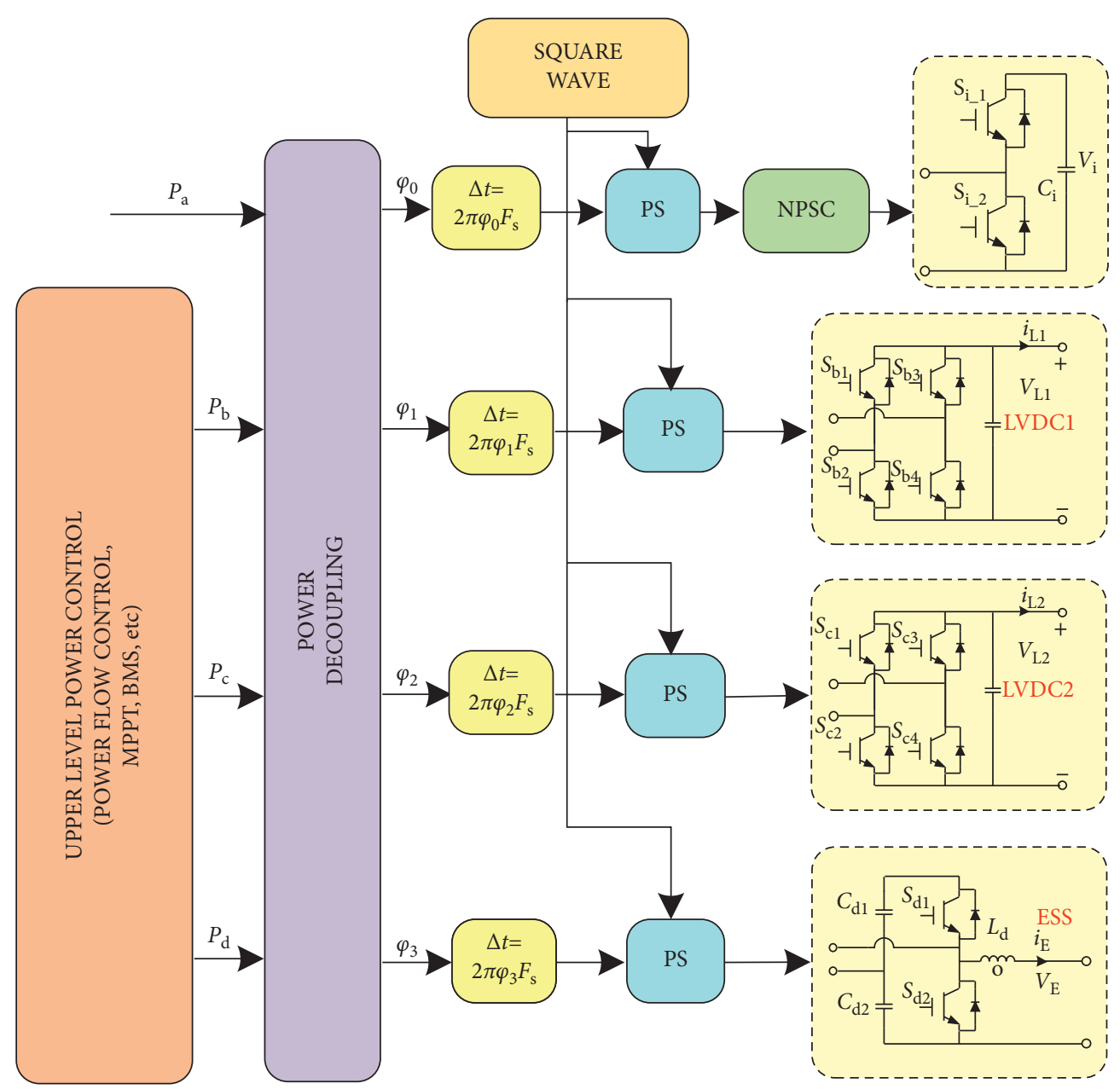

FIGURE 11: Control strategy of QP-M ${ }^{2}$ DC system.

TABLe 2: Simulation parameters.

Topological parameters

Parameters

MVDC voltage $(\mathrm{kV})$

Value

LVDC1 voltage $(\mathrm{V})$

20

LVDC2 voltage $(\mathrm{V})$

750

ESS voltage (V)

220

Number of MMC submodules

Value of SM capacitance (uF)

Phase-shift inductance $L_{b}, L_{c}, L_{d}(\mathrm{uH})$

Switching frequency $(\mathrm{kHz})$

Narrow phase-shift angle $\left({ }^{\circ}\right)$

TABLE 3: Working parameters of four modes.

\begin{tabular}{|c|c|c|c|c|}
\hline Port & Rated power $(\mathrm{kW})$ & Type & Rated power $(\mathrm{kW})$ & Type \\
\hline & \multicolumn{2}{|c|}{ Mode 1} & \multicolumn{2}{|c|}{ Mode 2} \\
\hline MVDC & 600 & Source & 100 & Load \\
\hline LVDC1 & 350 & Load & 350 & Source \\
\hline LVDC2 & 150 & Load & 50 & Load \\
\hline \multirow[t]{2}{*}{ ESS } & 200 & Load & 200 & Load \\
\hline & \multicolumn{2}{|c|}{ Mode 3} & \multicolumn{2}{|c|}{ Mode 4} \\
\hline MVDC & 407 & Load & 407 & Load \\
\hline LVDC1 & 350 & Source & 233 & Source \\
\hline LVDC2 & 57 & Source & 57 & Source \\
\hline ESS & 0 & Zero & 117 & Source \\
\hline
\end{tabular}




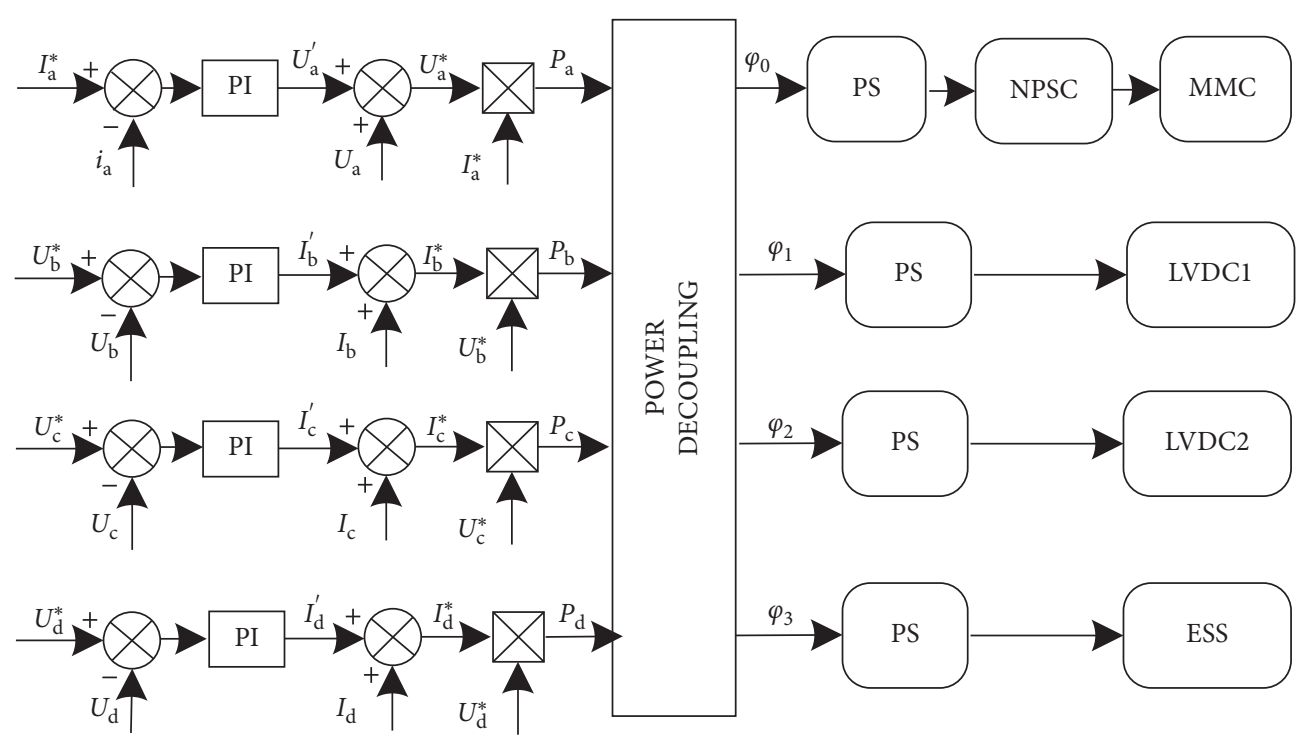

Figure 12: Simulation control strategy of QP-M ${ }^{2}$ DC system.

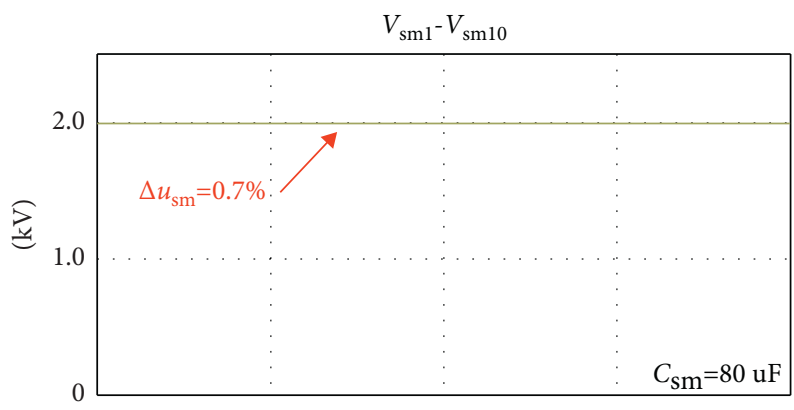

Time:[10 ms/div]

FIGURE 13: SM voltage waveform.
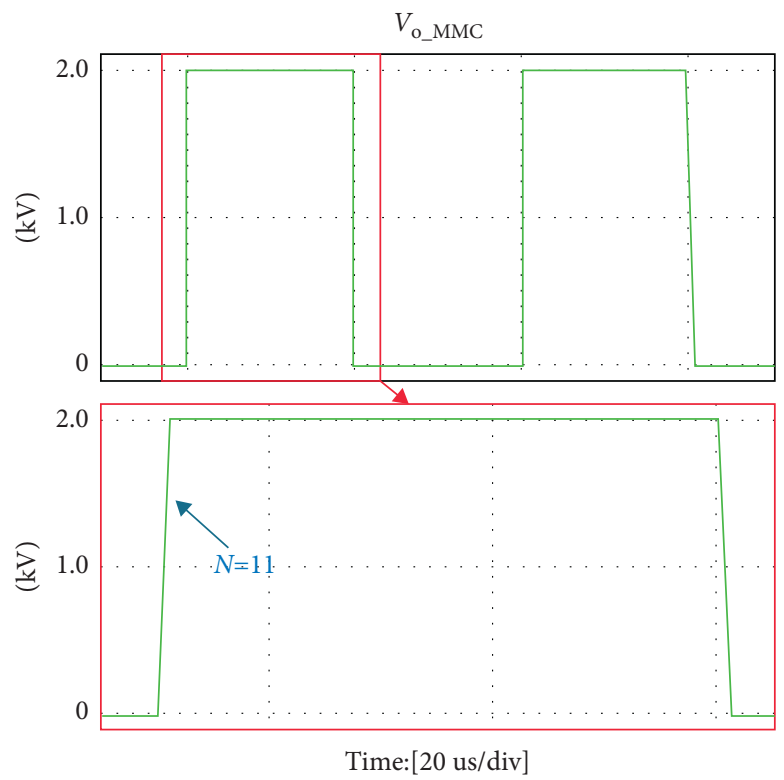

Figure 14: MMC side modulation voltage. 


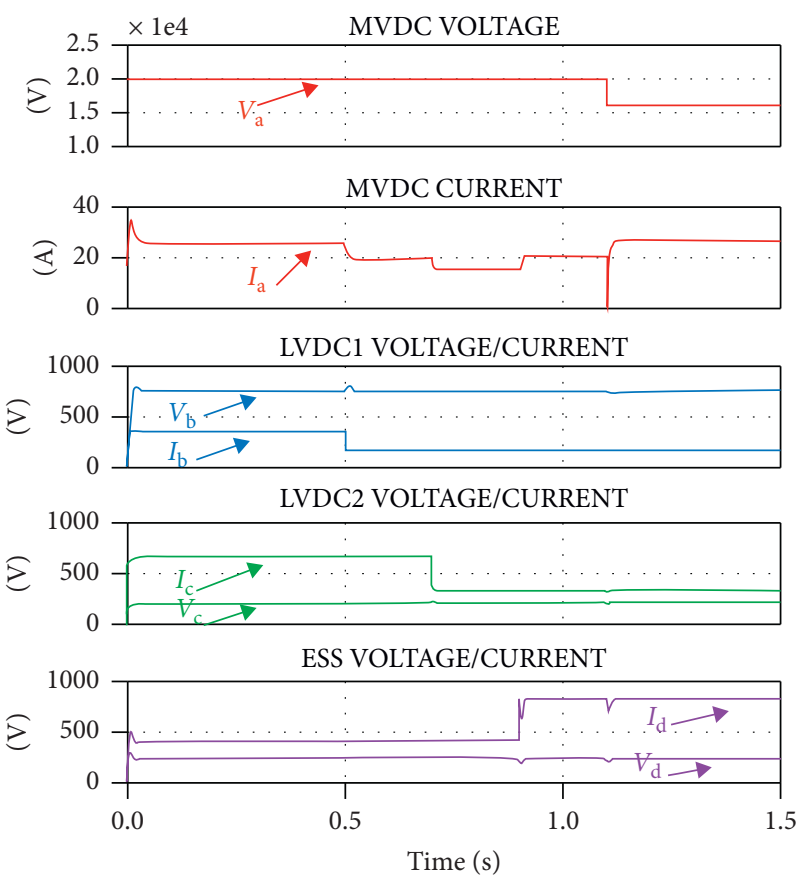

FIgURE 15: Mode 1 disturbance test waveforms.

MVDC port as the source, reducing the load of the LVDC1 port by $50 \%$ at $0.5 \mathrm{~s}$ and reducing the load by $50 \%$ of the LVDC2 port at $0.7 \mathrm{~s}$, increasing the load of ESS port from half load to full load at $0.9 \mathrm{~s}$, and reducing the voltage of MVDC port by $20 \%$ at $1.1 \mathrm{~s}$. From the simulation results, the port energy control strategy of QP-M ${ }^{2} \mathrm{DC}$ converter has good stability.

Figures $16-18$ are the HFL waveforms of the QP-M ${ }^{2} \mathrm{DC}$ converter operating in various modes. Figures 15 and 16, respectively, show the HFL waveform of one-source threeload mode when MVDC port and LVDC1 port are the sources. Figure 17 shows the HFL waveform from twosource one-load mode to three-source one-load mode. It can be seen that from simulation waveforms under various working modes the proposed energy control scheme has good performance.

\section{Experimental Results}

To verify the theoretical analysis, a down-scaled experimental prototype was built. Table 4 is the specific experimental parameters. dSPACE is selected as the platform controller, and the control mode is centralized control. The narrow phase-shift cyclic driving strategy, phase-shift control, and square-wave driving are realized in dSPACE, and then, the hardware dead time circuit on each submodule generates the switching signal with dead time, which is used to drive each submodule, full-bridge structure, and buck/ boost structure. The four phase-shift inductors are decoupled and integrated with two groups of EE cores. The isolation four-winding transformer is wound with UU magnetic core, and the power level is designed to be $2 \mathrm{~kW}$. The selection of switching devices at each port is shown in Table 5.
As shown in Figure 19, $V_{\mathrm{CH} 1}$ is the $750 \mathrm{~V}$ port voltage of QP-M ${ }^{2} \mathrm{DC}$ converter, $V_{\mathrm{CH} 2}$ is the $380 \mathrm{~V}$ port voltage, $V_{\mathrm{CH} 3}$ is the $220 \mathrm{~V}$ port voltage, and energy storage side $(48 \mathrm{~V})$ port voltage is $V_{\mathrm{CH} 4}$. The four ports of the QP-M ${ }^{2} \mathrm{DC}$ converter are all in steady state. The voltage waveform of MVDC is in the shape of step wave with an amplitude of $768 \mathrm{~V}$. The voltage waveform of LVDC1, LVDC2, and ESS is rectangular wave with a voltage amplitude of $375 \mathrm{~V}, 221.5 \mathrm{~V}$, and $47.5 \mathrm{~V}$, respectively. All ports work stably, which verifies the correctness of topology theory analysis.

Figure 20(a) shows the experimental waveform of QP$\mathrm{M}^{2} \mathrm{DC}$ converter at the start stage. During the transition from the prototype device starting process to no-load operation process, the dc voltage shock of each port does not exceed $5 \%$ of the rated voltage, the input current shock is not greater than $50 \%$ of the rated value, and the starting stage works normally. Figure 20(b) shows the waveforms of each port when the $380 \mathrm{~V}$ port of QP-M ${ }^{2} \mathrm{DC}$ converter switches the load. The $1.6 \mathrm{~kW}$ load is put into the $380 \mathrm{~V}$ port from no load, and then, the load is removed after a period of delay. In the figure, the voltage fluctuation value of each port is less than $5.3 \%$, and the maximum fluctuation is restored to stability after $294 \mathrm{~ms}$. The port capability control strategy of QP-M ${ }^{2}$ DC converter has good stability. Figure 20(c) shows the waveforms of each $220 \mathrm{~V}$ port in QP-M ${ }^{2} \mathrm{DC}$ converter when the load is switched off. The $120 \mathrm{~W}$ load is put into the $220 \mathrm{~V}$ port from no load, and then, the load is removed after a period of delay. In the figure, the voltage fluctuation value of each port is less than $1.9 \%$, and the maximum fluctuation is stabilized after $97 \mathrm{~ms}$. It reflects the stability of QP-M ${ }^{2} \mathrm{DC}$ converter when the 220 port is switched to load. Figure 20(d) shows the waveforms of each port in the charging process of the energy storage device of $\mathrm{QP}-\mathrm{M}^{2} \mathrm{DC}$ converter. In the experiment, the constant current charging instruction value 


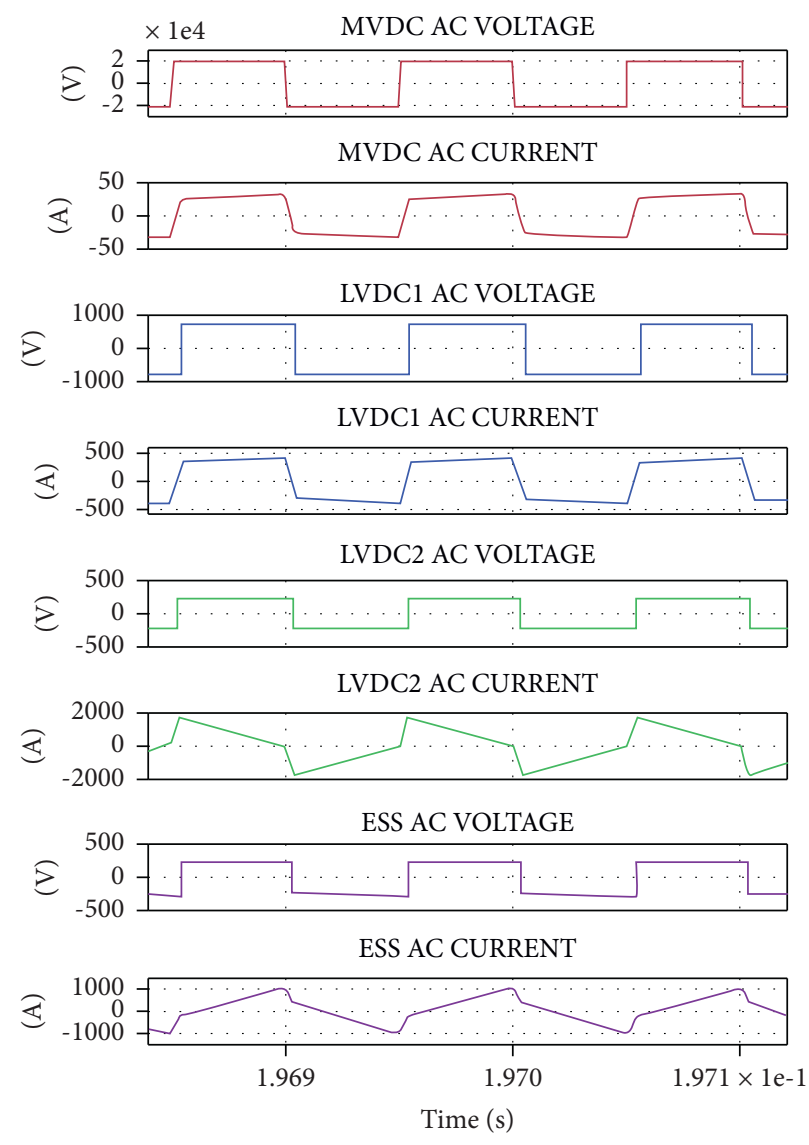

FIgURE 16: Mode 1 HFL waveforms.

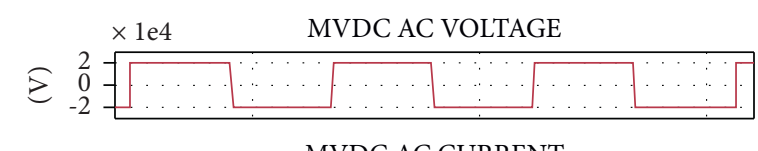

MVDC AC CURRENT
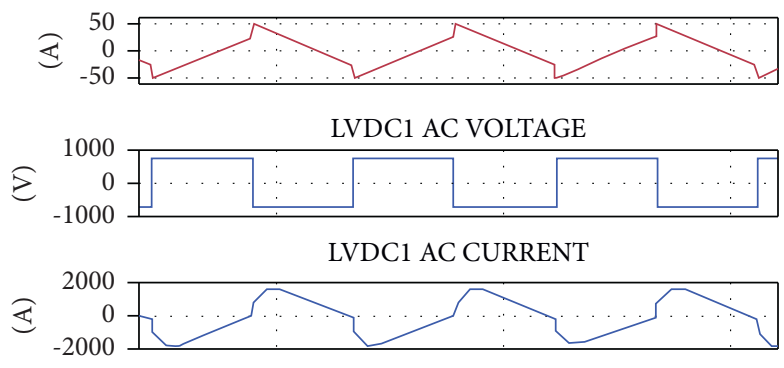

LVDC2 AC VOLTAGE

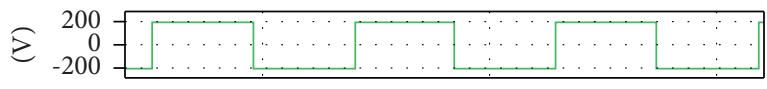

LVDC2 AC CURRENT

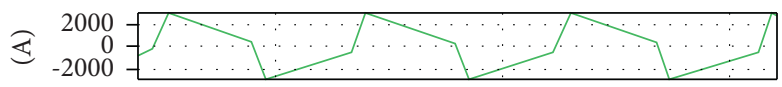

ESS AC VOLTAGE

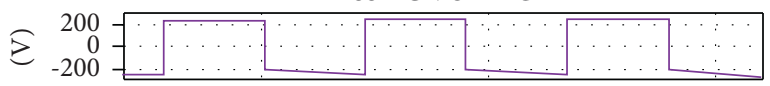

ESS AC CURRENT

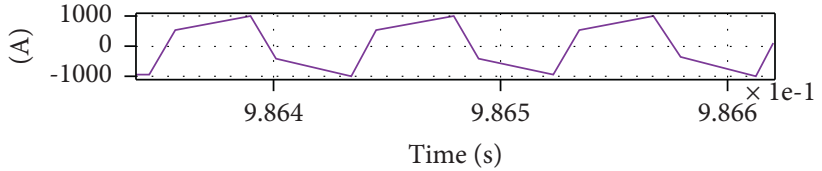

Figure 17: Mode 2 HFL waveforms. 


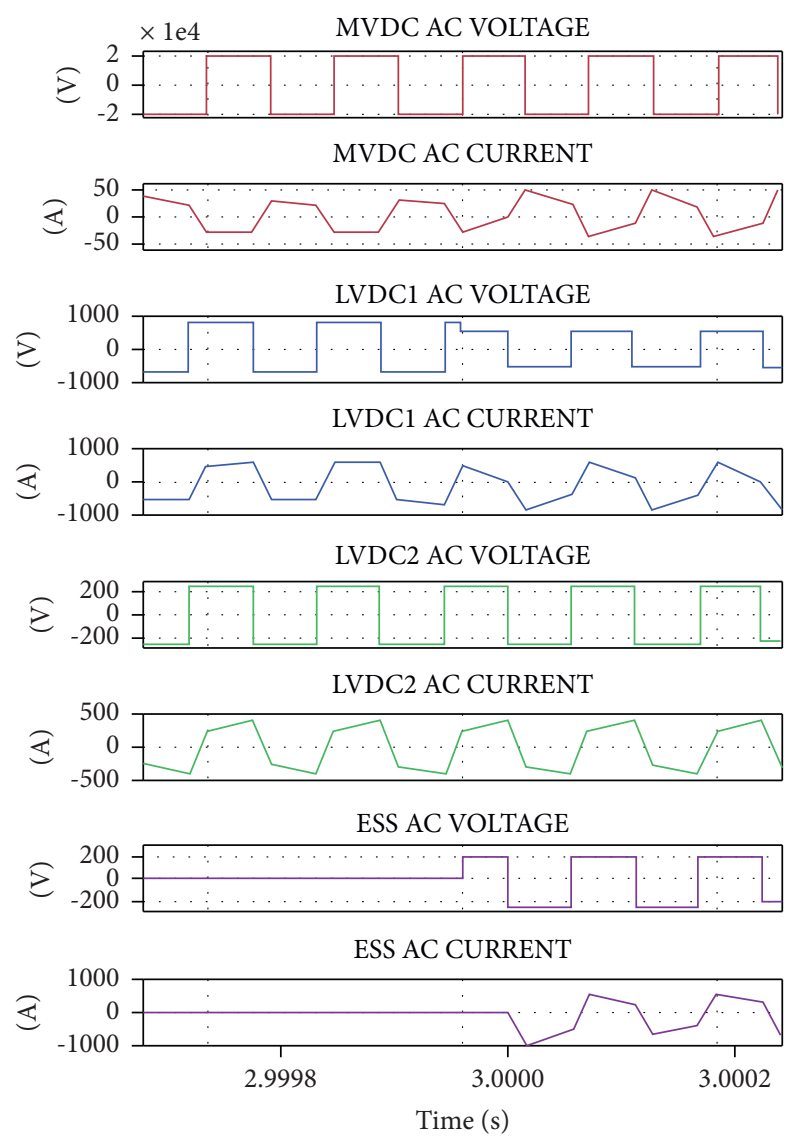

FIgURE 18: Mode 3 to mode 4 HFL waveforms.

TABLE 4: Experimental parameters.

Topological parameters

Parameters

MVDC voltage $(\mathrm{V})$

LVDC1 voltage $(\mathrm{V})$

LVDC2 voltage $(\mathrm{V})$

ESS voltage $(\mathrm{V})$

Number of MMC

Value of SM capacitance (uF)

Phase-shift inductance $L_{b}, L_{c}, L_{d}(\mathrm{uH})$

Switching frequency $(\mathrm{kHz})$

Narrow phase-shift angle $\left({ }^{\circ}\right)$

TABLE 5: Switch selection.

\begin{tabular}{lcr}
\hline Port $(\mathrm{V})$ & Type & Features \\
\hline 750 & FF225R17ME4 & $(1700 \mathrm{~V} / 225 \mathrm{~A})$ \\
380 & FF225R12ME4 & $(1200 \mathrm{~V} / 200 \mathrm{~A})$ \\
220 & IKP15N65F5 & $(650 \mathrm{~V} / 30 \mathrm{~A})$ \\
48 & IKW75N65RH5 & $(650 \mathrm{~V} / 80 \mathrm{~A})$ \\
\hline
\end{tabular}

changes from $1 \mathrm{~A}$ to $3 \mathrm{~A}$, and the voltage of each port runs stably. At this time, the converter works at a maximum power of $90 \%$, and the efficiency is about $96.1 \%$.

The efficiency of the four ports in three different working modes was tested, and the efficiency curves were drawn for three loads and one source, two loads and two sources, and one load and three sources, and results are shown in Figure 21. The full load efficiencies are $97.8 \%, 96.9 \%$, and $96.1 \%$, respectively, which proves that the proposed topology has high efficiency. 


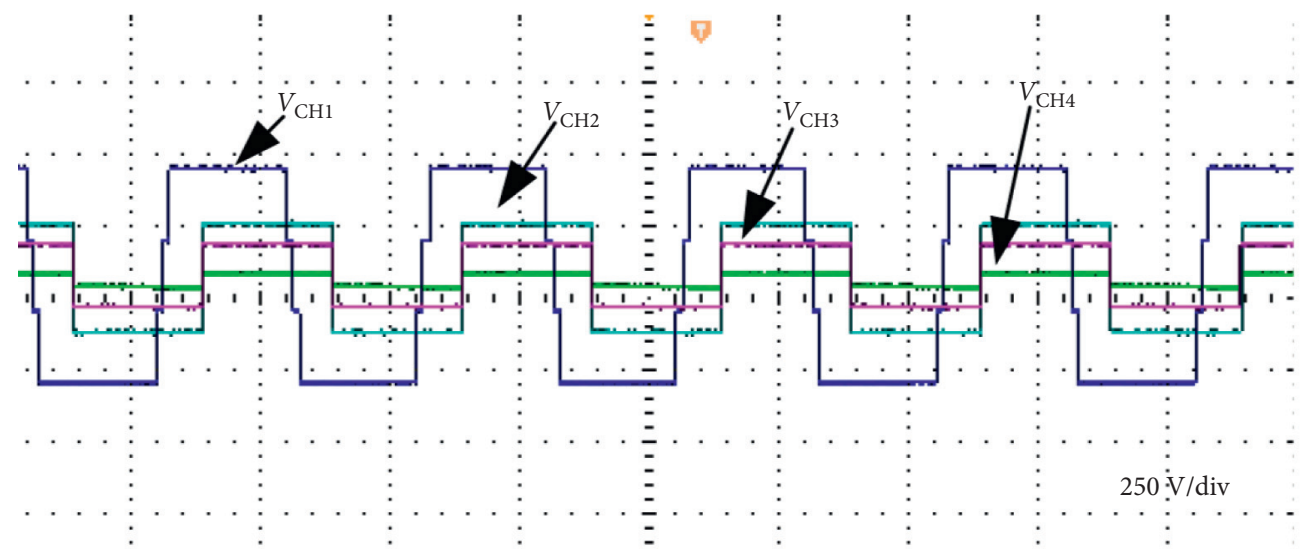

FIGURE 19: High-frequency link waveforms of each port.

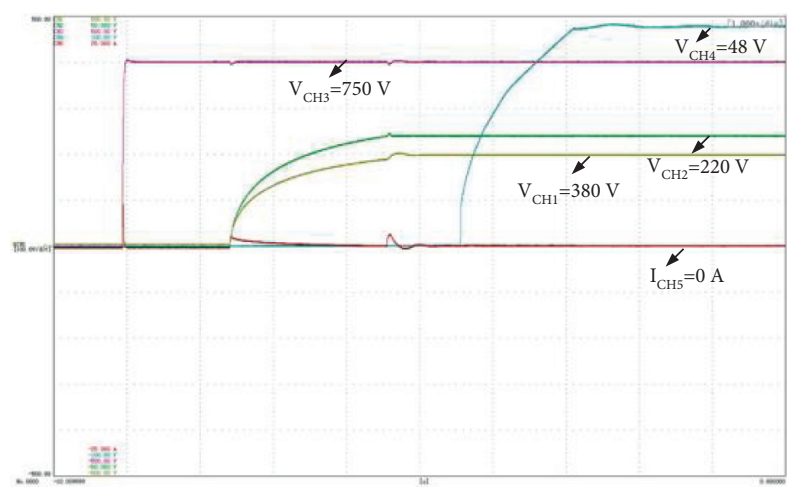

(a)

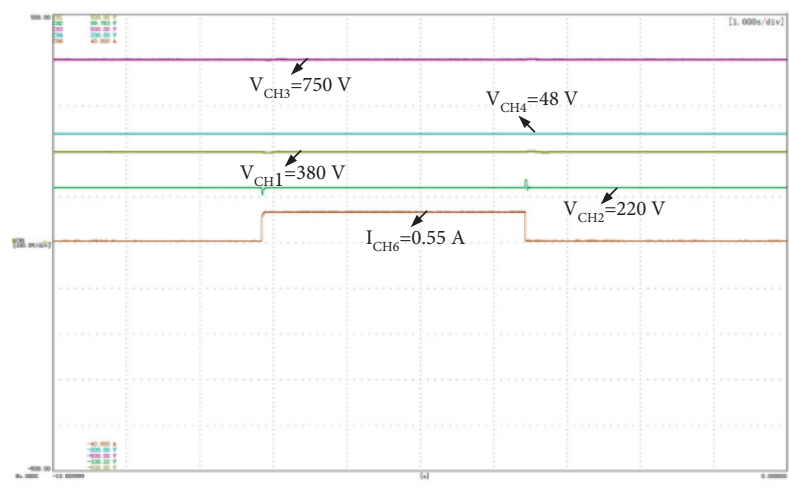

(c)

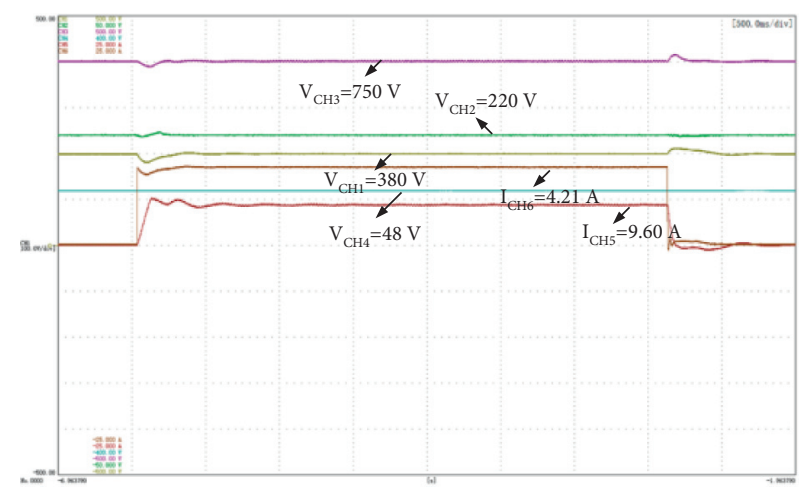

(b)

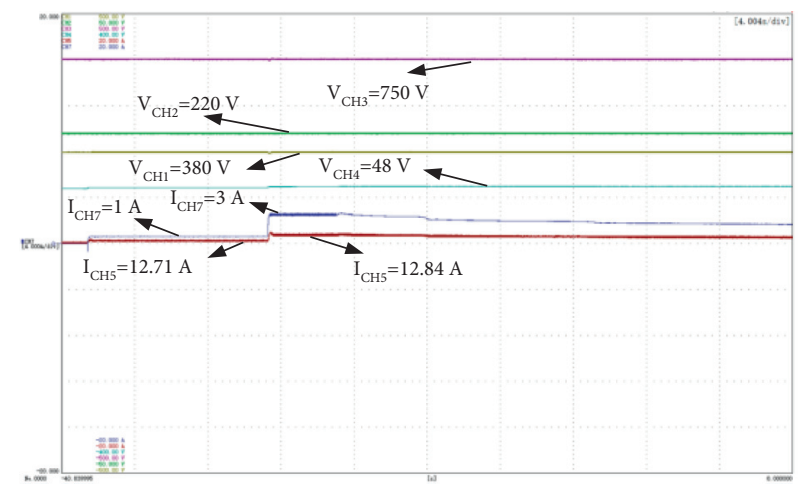

(d)

FiguRE 20: Experimental waveforms in different modes. (a) Start-up process. (b) Status switching of $380 \mathrm{~V}$ port. (c) Status switching of $220 \mathrm{~V}$ port. (d) Status switching of ESS port. 


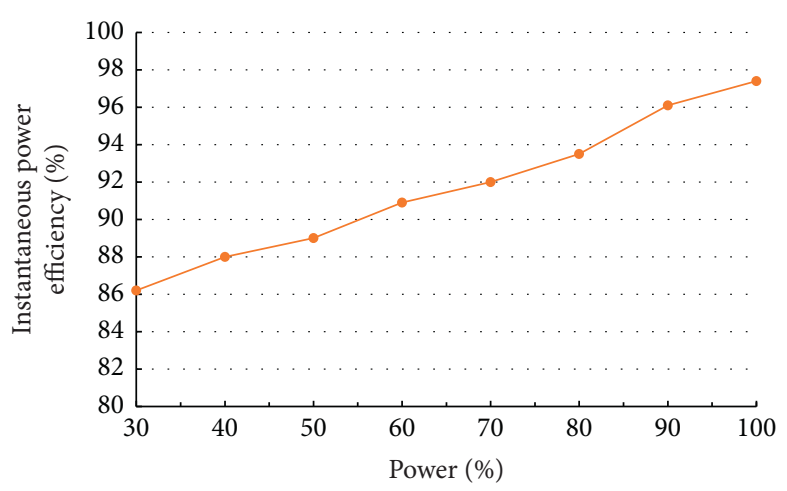

(a)

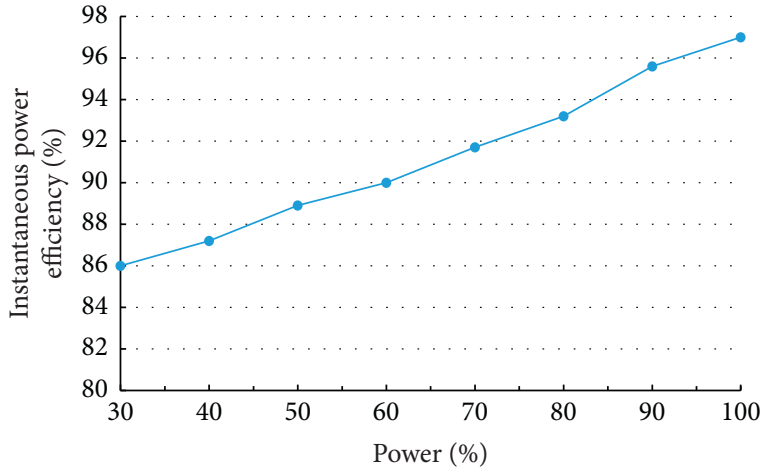

(b)

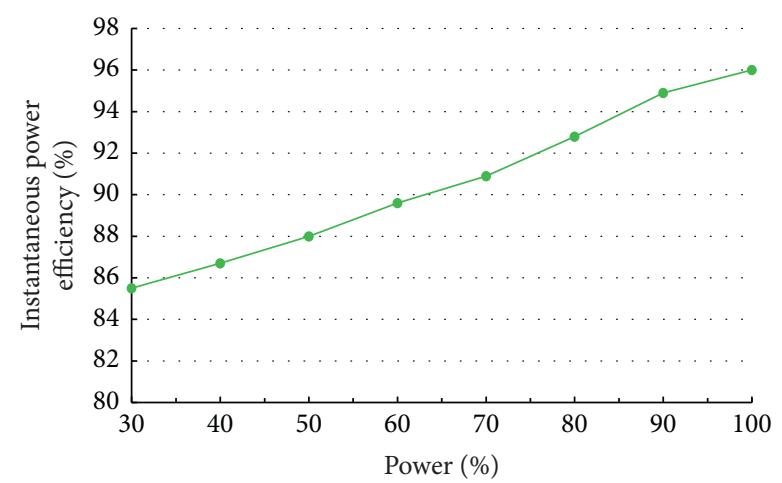

(c)

Figure 21: Efficiency curves for different working modes. (a) Efficiency curve of 1S-3L. (b) Efficiency curve of 2S-2L. (c) Efficiency curve of $3 \mathrm{~S}-1 \mathrm{~L}$.

\section{Conclusions}

In this study, a quad-port DC/DC converter (QP-M $\left.{ }^{2} \mathrm{DC}\right)$ topology scheme based on modular multilevel structure is proposed. Compared with the traditional multi-port power routing converter, this topology has advantages of high modularity, high flexibility, and high power density. Aiming at the modularization multilevel structure of medium- and high-voltage ports, this study proposes an NPSC modulation strategy, which is of great significance for the converter to reduce SM capacitance, which increases system power density and simplifies the equalization control. In this study, the topological structure, working principle, equivalent analysis of HFL, and power characteristics of QP-M $\mathrm{M}^{2} \mathrm{DC}$ are comprehensively introduced and analysed, the equivalent model of system control is established, and a control scheme of multiterminal energy routing converter is proposed. A set of QP- $\mathrm{M}^{2} \mathrm{DC}$ simulation systems was built by PLECS, and the simulation results verified the superiority of the proposed topology and the correctness and effectiveness of the proposed control scheme.

The simulation results show that in such a narrow phase-shift modulation strategy, the proposed topology can have stable operation in a variety of patterns, reduce the capacitance, and achieve better voltage balance at the same time. The experimental results show the converter efficiency of up to $97.8 \%$. It further shows the superiority of the proposed topology structure and the correctness and effectiveness of the proposed control schemes. In fact, there are some parts that need to be optimized. For example, there is room for further optimization of the design of medium-voltage high-frequency high-power transformer in the converter, including the treatment of insulation. Currently, optimization research is being carried out for this problem.

\section{Data Availability}

The data used to support the findings of this study were supplied by Jiaxun Teng under license and so cannot be made freely available. Requests for access to these data should be made to Jiaxun Teng (tengjiaxun@qq.com).

\section{Conflicts of Interest}

The authors declare that there are no conflicts of interest regarding the publication of this study.

\section{Acknowledgments}

This work was funded by the Science and Technology Project of State Grid Corporation of China "Research funding of energy Internet multi-energy flow integration and routing technology based on multi-port energy router" under Grant PD71-19-010. 


\section{References}

[1] L. Mariam, M. Basu, and M. F. Conlon, "A review of existing microgrid architectures," Journal of Engineering, vol. 2013, Article ID 937614, 8 pages, 2013.

[2] Y. Zhang, S. Wei, J. Wang, and L. Zhang, "Bus voltage stabilization control of photovoltaic DC microgrid based on fuzzy-PI dual-mode controller," Journal of Electrical and Computer Engineering, vol. 2020, Article ID 2683052, 10 pages, 2020.

[3] S. Choudhury, S. Nayak, T. P. Dash, and P. K. Rout, "Overview of DC microgrid as a conceivable future power system," in Proceedings of the 2018 Second International Conference on Inventive Communication and Computational Technologies (ICICCT), pp. 1541-1546, Coimbatore, India, April 2018.

[4] Q. Mei, W. Y. Wu, and Z. L. Xu, "A multi-directional power converter for a hybrid renewable energy distributed generation system with battery storage," in Proceedings of the 2006 CES/IEEE 5th International Power Electronics and Motion Control Conference, vol. 3, pp. 1932-1936, Shanghai, China, August 2006.

[5] M. Jafari, Z. Malekjamshidi, and J. Zhu, "A magnetically coupled multi-port, multi-operation-mode micro-grid with a predictive dynamic programming-based energy management for residential applications," International Journal of Electrical Power \& Energy Systems, vol. 104, pp. 784-796, 2019.

[6] X. Liu, Z. Zheng, K. Wang, and Y. Li, “An energy router based on multi-winding high-frequency transformer," in Proceedings of the 2016 IEEE Applied Power Electronics Conference and Exposition (APEC), pp. 3317-3321, Long Beach, CA, USA, March 2016.

[7] P. Frutos, F. Briz, A. Sanchez, and J. M. Guerrero, "QuadActive-Bridge as the basic cell of a MMC Based SST for der and DESS Integration," in Proceedings of the 2019 IEEE 28th International Symposium on Industrial Electronics (ISIE), pp. 2349-2355, Vancouver, BC, Canada, June 2019.

[8] D. Kirubakaran and R. R. Sathi, "Embedded controlled isolated bidirectional full-bridge DC-DC converter with flyback snubber," Advances in Power Electronics, vol. 2012, Article ID 730473, 10 pages, 2012.

[9] Y. Hayashi, Y. Matsugaki, and T. Ninomiya, "Fault tolerant operation of ISOP multicell DC-DC converter using active gate controlled $\mathrm{SiC}$ protection switch," Journal of Engineering, vol. 2016, Article ID 7345232, 8 pages, 2016.

[10] Z. Li, P. Wang, and Z. Chu, "Research on medium- and highvoltage smart distribution grid oriented power electronic transformer," Power System Technology, vol. 37, no. 9, pp. 2592-2601, 2013.

[11] B. Zhao, Q. Song, J. Li, Y. Wang, and W. Liu, "Modular multilevel high-frequency-link DC transformer based on dual active phase-shift principle for medium-voltage DC power distribution application," IEEE Transactions on Power Electronics, vol. 32, no. 3, pp. 1779-1791, 2017.

[12] R. Mo, H. Li, and Y. Shi, "A phase-shifted square wave modulation (PS-SWM) for modular multilevel converter (MMC) and DC transformer for medium voltage applications," IEEE Transactions on Power Electronics, vol. 34, no. 7, pp. 6004-6008, 2019.

[13] Y. Chen, S. Zhao, Z. Li, and Y. Kang, "Multi-object control of an isolated DC-DC modular multilevel converter," IET Power Electronics, vol. 11, no. 8, pp. 1338-1349, 2018.

[14] S. Shao, M. Jiang, J. Zhang, and X. Wu, "A capacitor voltage balancing method for a modular multilevel DC transformer for DC distribution system," IEEE Transactions on Power Electronics, vol. 33, no. 4, pp. 3002-3011, 2018.

[15] C. Sun, J. Zhang, X. Cai, and G. Shi, "Voltage balancing control of isolated modular multilevel dc-dc converter for use in dc grids with zero voltage switching," IET Power Electronics, vol. 9, no. 2, pp. 270-280, 2016.

[16] G. Sha, Q. Duan, W. Sheng et al., "A multiport electric energy routing scheme Applied to battery energy storage system," Journal of Engineering, vol. 2021, Article ID 6637926, 15 pages, 2021.

[17] Y. Tian, Z. Chen, F. Deng, X. Sun, and Y. Hu, "Coodinative control of active power and DC-link voltage for cascaded dual-active-bridge and inverter in bidirectional applications," in Proceedings of the 2014 IEEE Energy Conversion Congress and Exposition (ECCE), pp. 2249-2256, Pittsburgh, PA, USA, September 2014.

[18] J. Teng, X. Sun, Z. Bu, W. Zhao, and X. Li, “Optimization scheme based on high-frequency link interconnection of submodules," IEEE Transactions on Power Electronics, vol. 36, no. 12, pp. 13645-13659, 2021.

[19] A. Q. Huang and J. Baliga, "FREEDM System: role of power electronics and power semiconductors in developing an energy internet," in Proceedings of the 2009 21st International Symposium on Power Semiconductor Devices and IC's, pp. 9-12, Barcelona, Spain, June 2009.

[20] X. Sun, J. Teng, L. Qi, Y. Pan, Z. Bu, and M. Zhang, "Research on triple-port MMC-based PET scheme based on high frequency links interconnection of horizontal sub-modules," in Proceedings of the 2020 IEEE 9th International Power Electronics and Motion Control Conference (IPEMC2020-ECCE Asia), pp. 2061-2067, Nanjing, China, November-December 2020.

[21] T. Zhao, G. Wang, S. Bhattacharya, and A. Q. Huang, "Voltage and power balance control for a cascaded H-bridge converter-based solid-state transformer," IEEE Transactions on Power Electronics, vol. 28, no. 4, pp. 1523-1532, 2013.

[22] Z. Bu, J. Teng, X. Sun, Y. Pan, and Y. Pan, "Low frequency voltage ripples decoupling with switched-capacitor conversion for an MMC-based SST," IEEE Transactions on Industrial Electronics, 2021, In press.

[23] X. Sun, J. Teng, Z. Bu, Y. Pan, W. Zhao, and X. Li, "Research on triple-port SST scheme based on the natural elimination of MMC sub-modules voltage fluctuation and imbalance," IEEE Journal of Emerging and Selected Topics in Power Electronics, 2021, In press.

[24] T. Sun and X. Pei, "Comparison analysis on EMI sources of MMC under NLM and CPS-PWM strategies," in Proceedings of the 2019 4th IEEE Workshop on the Electronic Grid (eGRID), pp. 1-4, Xiamen, China, November 2019.

[25] L. He, Z. Shuai, and J. Shan, "Contrastive analysis of multicarrier modulation strategy for modulation multi-level converter," Journal of Power Supply, vol. 17, no. 5, pp. 56-64, 2019. 